\title{
Application of the Rain-flow Counting Method in Fatigue
}

\author{
Guojun Liu ${ }^{1, a}$ Dahuan Wang ${ }^{1, b}$ and Zeyou Hu ${ }^{1, c}$ \\ ${ }^{1}$ College of Civil Engineering, Sichuan Agricultural University, China \\ a93304276@qq.com, b1664792318@qq.com, '123053841@qq.com
}

Keywords: The rain flow count method, principle, development, application, fatigue

\begin{abstract}
In the statistics of stress-strain cyclic response involved with cyclic-loading, rain-flow counting method has always been thought the most reasonable way to proceed cycle counting, based on which researchers could conduct fatigue test or calculate the damage extent according to $\varepsilon-\mathrm{N}$ (or $\mathrm{S}-\mathrm{N}$ ) curve. That is the main reason why this method is widely used. This article will minutely discuss the principle, developing status, computer-based realization and application of rain-flow counting method, after which briefly discuss its application in fatigue problem in road and bridge.
\end{abstract}

\section{Introduction}

In engineering practice, the work loading imposed on structure varies with operating condition and service environment, belonging to stochastic loading. Stochastic loading is a kind of irregular dynamic loading, changing with time. When analyzing the fatigue damage, people normally describe stochastic loading by using statistic-analysis method. Counting method and power spectral method are the most widely used ways used in the analysis.

There is a great variety of counting method, such as peak-counting range-counting, apices-crossing and rain-flow counting. Different ways of counting produce disparate outcomes, even for the same statistical object during the same period of time. The normally used counting method could be categorized into two types: single parameter counting method and double parameter counting method. Single parameter counting only observes one parameter in loading cycle, visualized and uncomplicated, but lacking strictness and accuracy. In this way researchers could not get the information about the change of loading frequency and the sequence of loading occurrence. Also, it is not capable of depicting cycle performance of the loading, due to which, it is merely an approximate method for counting .However, double parameter counting is able to record two parameters in loading cycle. Compared with that of single parameter counting, the output of double parameter counting is much more comprehensive, thus being a preferable way for counting. Double parameter could be categorized into rain fall method, apical-range method and apical-average method.

Both loading amplitude and loading frequency index are important parameter in structural fatigue calculation. Amplitude and frequency are also the main reason for fatigue damage, thus requiring counting method for the statistics. Rain fall counting is processed with the stress-strain behavior of material, taking into account the non-linear relationship of stress and strain. Therefore, the gained loading cycle is consistent with the stress-stain hysteresis loop and meanwhile, it is able to precisely mirror material's fatigue damage. Since this counting method could be conducted on computer platform, it could be automated and programmed without much effort. In contrast to other counting methods, rain-fall counting is more accurate, so it is extensively applied in the research and application of fatigue-related problems.

\section{The Rain-flow Counting Method}

Basic Principle. The rain flow count method is a method of double parameters count proposed by Matsuiski and Endo, in which stress-strain behavior was taken into account in the $1950 \mathrm{~s}$, count results with stress amplitude and mean stress two parameters to describe the stress effect of cyclic loading. They pointed out that the existence of the plastic material was the necessary condition of fatigue damage and stress-strain cyclic hysteresis loop (figure 1) was the major form of plastic 
materials. And a complete cycle quantified as a fatigue damage, while assuming that the process of drastic change damage would not be small hysteresis curve affected. This actually reflected the memory of the material properties, and this feature was a key property to validate the rationality of the cycle counting method. Memory characteristic means: a big stress aborted by a small cycle still remembered the original path. Specifically shown in figure 2, it was assumed to be loaded first by $\mathrm{O}$ to 1 , and then unloaded to 2 . While if we load and reload the value of more than 1 point, the stress strain response would along the curve of 2-1-3, rather than along the curve of 2-l'-3' to reach on the dotted line in the figure 3' point. (0-1-3 for cyclic stress strain curve, 2-1-3' for the hysteresis loop curve).

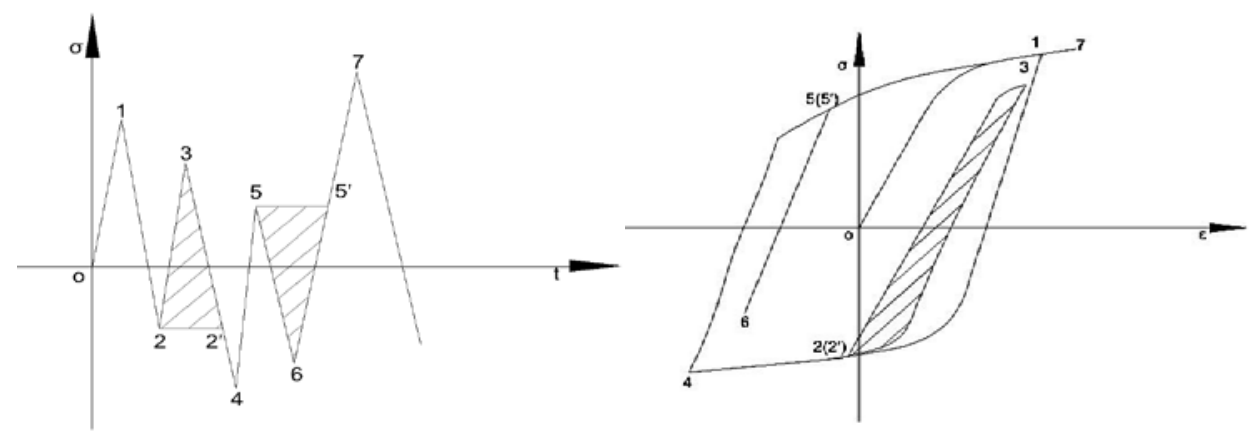

Fig. 1 Stress-strain hysteresis curve

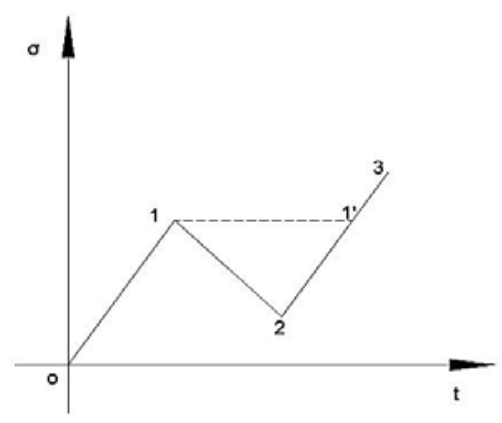

a) Stress - time history curve

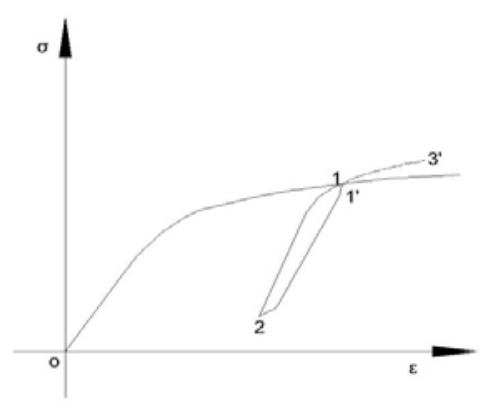

b) The stress-strain response curve

Fig. 2 Memory characteristics

Counting Rules. Rain flow counting method is also called overhead method. First, stress-time curve of axis is inverted $90^{\circ}$ so that the timeline is downward, then taking the following counting rules.

(1) The starting point of the rain flow in the test record, that is, from 1, 2, 3, and so on. All of them are sharp point.

(2) The rain flow in the flow to the peak (roof) under the vertical drop, has been flowing to the opposite one which is larger.than at the beginning of absolute value.

(3) When rain flows encountered rain from above the roof of the shed, it stops flowing, and this constitutes a loop.

(4) According to the beginning and end of the rain flow, draw various circulation, remove all the circle one by one, and record the peak value.

(5) The level of each rain flow length can be used as the cyclic stress amplitude, and average value of peak valley is the mean stress.

\section{Algorithm Model of Rain-flow Counting Method}

All sorts of rain-flow counting model which have been put forward at present are finding out the hysteresis loop in cycle stress cycle for the principle. After the authors' analysis and finishing, now 
more mainstreaming and scientific algorithm mainly includes four peak valley rain counting method, three metamorphoses counting method, the rain flow count method based on recursive algorithm, improving the rain flow method counting model to real-time counting model, and so on.

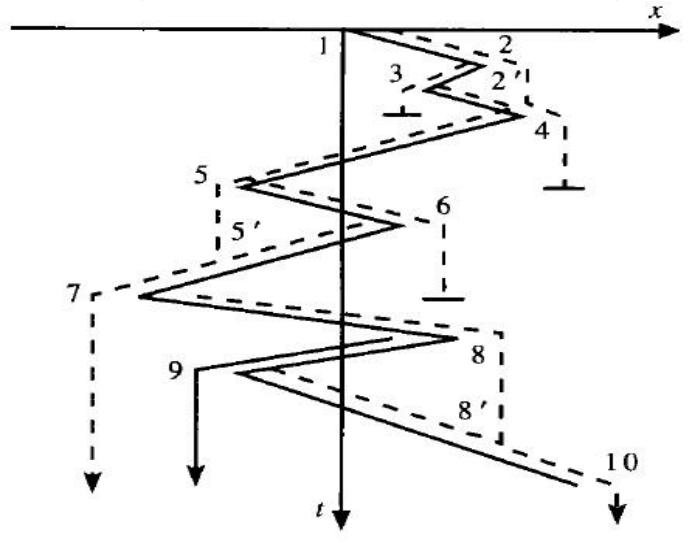

Fig. 3 Rain-flow counting method

Four Peak Valley Rain Counting Method. Literature [1] based on four peak valley rain flow counting method introduced the rapid implementation method of rain flow counting. Before the count, this method requires to handle the load time history first that wiping off the peak value and adjusting the course process. The data in the process of realizing procedure needs to be kept independently, and the selection of count decision point follow the principle of One-way selection and marching circularly. Gradually carrying forward until let the load - time history analysis become a series of full cycle processing.

Three Metamorphoses Counting Method. Jiang Hui et al discussed the main count principles in the process of loading course count and pointed out this principle's advantages that compared with other principles.

Advantages: "Three changes procedure" counting principle with graphical method on the principle of rain flow truncation principle as well as the "four point" in the peak valley counting value is consistent. Therefore, the correctness of the count is guaranteed. In addition, it is one of the principles of adopting the variable to count the various methods, more visual, at the same time easy to understand. So the difficulty of programming is reduced. Disadvantages: we need to do secondary statistics for convergent-divergent wave.

The Rain-flow Counting Method of Recursive Algorithm. Jiang Dongfang proposed the rain flow count recursive algorithm. Firstly, the paper indicated rain flow counting method about fatigue analysis was equivalent to another explanation, and recursive algorithm of rain flow counting method was proposed which was based on analyzing interpretation. This algorithm is based on two valley points as a boundary. Containing more than one peak stress point load time history is divided into three sub-segments, and each sub-segment continued to be broken down into three sub-segments shorter by using recursive algorithm until each sub-segment contains only one peak point or does not contain a peak point. Any final decomposition contains only one peak point of a sub-segment sub-cycle on a map that you can simultaneously calculate maximum and minimum value in a recursive calculation process. The advantage of this algorithm is that the algorithm does not require repeated culling peak value point of the local sub-cycle refresh data after load-time course work, while being able to retain the information sub-recurring order. And the first algorithm the programming is extracted maximum stress cycles. Its disadvantages are: the algorithm is not suitable for real-time fatigue damage calculation and implementation of the algorithm will take up more storage space.

Improved Rain-flow Method Real-time Calculation Model. Literature [2] pointed out that the traditional model of rain flow counting method was divided into closed-end calculation model and real-time counting model. Count fully enclosed model is a counter model which was developing overseas in the 1960s, and this disadvantage is that we can process after all the data collected, which can not achieve real-time processing. Rain flow method and real-time count model overcame the limitation of closed counting model that reproduced the stress time histories before counting. And it 
does not need to readjust or docking before counting statistics stress time histories, but counted directly on the peak value of the real stress histories appearing sequentially. Rain flow method real-time count model can improve data processing efficiency. But in the second stage of the process but it does not take into account how to ensure whether there is no any leakage points after the convergence wave docking with the divergent wave. So, in the literature [2], adding flow a docking algorithm solved the data conversion and docking problems in the data processing, while ensuring the reliability of data docking and the data processing efficiency.

Reflection on Different Computing Models. Above several rain flow counting methods are now in-depth and thorough study of the rain flow algorithm models, which are mentioned improved algorithm model on the basis of the basic principles of the rain flow counting, in order to solve or make up for model drawbacks of different application areas and the initial rain flow counting algorithm. So they have their own advantages and range of applications. It should be selected particularly in engineering applications or research according to the actual situation.

\section{Implementation of Rain-flow Counting Method in the Program}

The main function of rain-flow counting method is that the measured load history data which has been removing the detection value of peak valley and the invalid amplitude is expressed in the form of discrete load cycle. Each step processed by the computer requires programming. But the most basic programming process mainly includes the following links:

Data Compression. Data compression: 1) Detection of peak and valley; 2) Removal of invalid amplitude. These amplitudes are so small in many fatigue life cycles that they can be ignored. In order to simplify the load process wave model, it is necessary to remove the invalid amplitude. There are many physical models of removal of invalid amplitude, which can be selected according to the nature of the data and the cyclic wave form characteristics of the studied objects.

Extraction of the Number of Cycles. It includes the three steps: a rain-flow counting, docking and two rain-flow counting. However, it needs to be determined by the different waveform in the actual situation. For example: the high value of skewness waveform can be only completed by once the rain-flow count. At present, the usual programming software is MATLAB, etc. Many fatigue analysis software provides program interface of Matlab or integrate the algorithm directly into the software such as NSoft and so on. Because of a large amount of data collected, we need to use the database to manage the data, which is required for the database management such as SQLserver2000.

\section{Application of Rain-flow Counting Method in Fatigue Problem}

A variety of algorithms are based on the principle of the basic rain flow counting. Because it combines the stress-strain hysteresis loop and fatigue damage and pluses the advantage in programming processing, the rain-flow counting method has been widely used in the problem of fatigue load and damage, which involves a large number of cyclic load data.

Application of Structural Fatigue Life Estimation. The application of rain-flow counting method in fatigue life estimation was analyzed in the literature [3]. The method introduced in this literature can be summarized as: continuous 3 points determine the peak and valley and continuous 4 points record full wave. The rest half wave is connected to the full wave, and then this method simplify the counting process. Finally, according to the working frequency and the fatigue life under different amplitude loads, the fatigue life of the component under certain conditions is estimated by using the Miner linear cumulative fatigue damage criterion.

Vehicle Fatigue Damage Analysis. Rain flow count method in the fatigue performance and durability of the vehicle has been widely used. Predictably, it will become one of the hot issues of domestic research in the future for a long time in China. Sun Li et al reviewed analysis the rain flow count method of the theory and application, the main contents including: basic theory study and the counting method application in vehicle parts fatigue damage.

Statistical Analysis of Vehicle Load Spectrum. Zhang Zhenmiao et al adopted the rain flow count method for freight train coupler loads measured and time to statistical count, establishing the 
amplitude and mean as the phalanx of double parameters data table. In order to facilitate statistical analysis, the authors also established with the frequency histogram, in which the abscissa denotes the variation range. The above work provides ample data for preparation of coupling load spectrum. To remove invalid amplitude principle, the author put forward a new invalid amplitude of judging standard. The author thought omit standard for invalid amplitude should use material of the threshold value of delta $\triangle K_{\mathrm{tb}}$ to calculate, which appearing more convincing and theoretical basis.

The principle of rain flow count method was applied in Zhao Xiaopeng's research. A certain type of suvs load spectrum was measured in a certain range to strengthen road load, on the basis of which, first of all, authors preprocessed to the load spectrum. Then load spectrum of compression, extrapolation, superposition and the reconstruction of the time-domain application instance based on the rain flow count method were given. The paper showed the rain flow count method could keep the the time course of the compression load spectrum under the condition of original load spectrum fatigue damage, preparing for a laboratory road simulation test for the next step.

\section{Conclusion}

Based on above-mentioned analysis, we could draw a conclusion that at present, rain fall counting is widely applied in researches and application involved with the assessment of fatigue damage and fatigue endurance. The applied field is mainly concentrated on the fatigue damage of mechanical part, formulation of vehicles' loading spectrum, aeromechanic fatigue and the research of railway defects and fatigue endurance. Currently, this method is less used in the application and research of road bridge affected by the vehicle loading. Following China's economic development, the transportation pressure is growing rapidly, so are fatigue problems involved with road bridge affected by vehicle loading .It is an urgency to find the fatigue stress cycle that greatly harm the road/bridge's structure among complicated and stochastic vehicle loading. Therefore, based on natural science fund projects

《Research on Fatigue Damage Model for Concrete Based on Residual Strain and Failure load model of vehicles》 conducted by Doctor Liu Guojun, the thesis suggests that it is reasonable to adopt the four peak valley method of rain-fall counting to count the fatigue frequency of road bridge affected by vehicle loading, and thus laying the caculating foundation for the modeling of vehicle loading.

The application of rain flow count method in the project mainly includes the following two links:

(1) Measured vehicle loads on structures under the action of stress history is filtered, such as low-pass filtering, eliminating singular item and trends;

(2) In the whole session, I believe that how to set the abnormal value criterion about course wave of the stress response and how to design invalid amplitude threshold in the pretreatment process have certain research value.

\section{Acknowledgments}

This work was supported by a grant from the National Natural Science Foundation of China, whose name is "Research on Fatigue Damage Model for Concrete Based on Residual Strain and Failure load model of vehicles" (No. 51408385).

\section{References}

[1] Zhou Jun, Tong Xiaoyan. Rapid realization method of rain-flow counting[J]. Science Technology and Engineering, 2008, 13(8):3544-3548.

[2] Tian Jun, Li Qiang. Improved model of rain-flow real-time counting method[J]. Journal of Beijing Jiaotong University, 2009, 01:28-31.

[3] Wang Hongwei, Xinbo, Luo Hongyun. The rain-flow counting method and its application in fatigue life estimation[J]. Mining \& Processing Equipment, 2006, 03:95-97. 